Revista de Psicología Vol. 30 (1), 2012 (ISSN 0254-9247)

\title{
Predictores del rendimiento académico en adolescentes con disposiciones resilientes y no resilientes ${ }^{1}$
}

\author{
José Gaxiola Romero², Sandybell González Lugo ${ }^{3}$, \\ Zita Contreras Hernández ${ }^{4}$ y Eunice Gaxiola Villa ${ }^{5}$ \\ Universidad de Sonora, México
}

Existen factores de riesgo que afectan el rendimiento académico de los adolescentes de preparatoria, estos pueden ser contextuales e individuales. La resiliencia es un fenómeno que implica la superación de los riesgos que se enfrentan. El objetivo del estudio fue probar un modelo hipotético de ecuaciones estructurales en dos grupos de estudiantes de preparatoria, uno de resiliencia y otro de no resiliencia, para evaluar los efectos que tienen las variables contextuales, las metas y la autorregulación sobre el rendimiento académico. Los resultados indican diferencias en las variables que explican el rendimiento de ambos grupos.

Palabras clave: resiliencia, rendimiento académico, adolescentes, factores de riesgo.

Predictors of academic achievement in adolescents with dispositions to resilience and non-resilience

There are risk factors influencing academic achievement in high school adolescents that can be contextual and individual. Resilience is the ability of individuals to cope with challenging life experiences. The study aimed to prove an hypothetic model of structural equations in two groups of high school students, a dispositional resilience group and a non-dispositional resilience group, to assess the effects of contextual variables, goals and self-regulation on academic achievement. Results show differences in the variables that explain academic achievement in both groups. Key words: Resilience, academic achievement, adolescents, risk factors.

1 Esta investigación se desarrolló gracias al apoyo a los proyectos de investigación convocatoria PROMEP 2010-2011.

2 Doctor en Ciencias Sociales. Profesor investigador del Departamento de Psicología de la US. Miembro del Sistema Nacional de Investigadores de México. Cuenta con publicaciones a nivel nacional e internacional sobre resiliencia. Contacto: Matemáticos 5, C. P. 83240, Hermosillo, Sonora, México; joegaxiola@gmail.com

3 Licenciada en Psicología. Trabaja en proyectos de investigación en el área de resiliencia. Ha realizado ponencias a nivel internacional y nacional, además de publicaciones de artículos y el capitulo de un libro. Contacto: Av. San Hiram 71, C. P. 83179, Hermosillo, Sonora, México; sandybell.gonzalezl@gmail.com 

El logro educativo de los estudiantes de preparatoria en México es insuficiente. A nivel nacional, de acuerdo con los datos oficiales obtenidos de los Exámenes de Evaluación Nacional del Logro Académico en Centros Escolares ENLACE (SEP, 2010a), en el nivel medio superior el $11.5 \%$ de los evaluados obtuvieron en habilidad lectora un nivel de dominio insuficiente, el 31.2\% fue elemental, el $47.7 \%$ bueno, y solamente el 9.6\% resultó excelente. Por otra parte, en habilidad matemática el $40.6 \%$ del total de alumnos evaluados obtuvieron un nivel de dominio insuficiente, el $39.1 \%$ elemental, el $15.1 \%$ bueno y únicamente el 5.3\% excelente. Para el Estado de Sonora, el 78.6\% de los estudiantes resultaron con grado insuficiente y elemental en matemáticas, y en habilidad lectora el 45.1\% resultaron también insuficientes (SEP, 2010b).

Los datos seńalados muestran que gran parte de los estudiantes de preparatoria no poseen las habilidades básicas necesarias para su desarrollo académico, motivo por el cual resulta pertinente el estudio de las variables asociadas al logro académico en estudiantes de educación media superior, a fin de incidir sobre dichos aspectos y generar una mejora en su aprovechamiento para aumentar sus probabilidades de ingreso a la universidad. Uno de los indicadores asociados al logro académico de los alumnos lo constituye su nivel de rendimiento medido con el promedio de calificaciones ${ }^{6}$. El rendimiento académico puede

4 Licenciada en Psicología, enfocada en la investigación de la resiliencia en jóvenes. Ha participado en ponencias nacionales e internacionales, así como en publicaciones en revistas científicas. Contacto: Av. 14 de Abril 178, C. P. 83190, Hermosillo, Sonora, México; zitagpe@gmail.com

5 Licenciada en Psicología. Participa en la Maestría en Ciencias de la Salud de la US. Cuenta con publicaciones en revistas científicas y ponencias a nivel nacional. Contacto: Matemáticos 5, C. P. 83240, Hermosillo, Sonora, México; ladyunic@gmail.com

6 El logro académico es un constructo general que requiere ser medido con indicadores tales como habilidades y aptitudes mediante pruebas estandarizadas (De Hoyos, Espino \& García, 2010). En la presente investigación se utilizará el rendimiento académico evaluado a través de las calificaciones, como un indicador específico del logro académico. 
definirse como el nivel de conocimiento demostrado en un área o materia comparado con la edad y el nivel académico (Jiménez, 2000), y constituye una medida de la capacidad de respuesta de la persona, que expresa de manera estimativa lo que ha aprendido como resultado de un proceso de enseñanza (Pizarro \& Clarck, 1998).

En la mayoría de universidades de México, uno de los requisitos de ingreso lo constituye el promedio general con que egresan los estudiantes del nivel medio superior. El promedio de calificaciones es el indicador más utilizado para medir el rendimiento académico, ya que refleja el conocimiento del alumno en relación con ciertas materias (Cascón, 2000). Entonces, aquellos egresados con mayor promedio, tendrán mayor posibilidad de ser aceptados en el nivel educativo superior.

La escuela preparatoria en México es cursada durante los ańos de la adolescencia (15 a 17 años aproximadamente). La adolescencia se entiende como un proceso de transición entre la infancia y adultez (Frydenberg, 1997), caracterizada por un período de cambio, crecimiento y desequilibrio que funciona como puente entre dos etapas estables de la vida (Vargas \& Barrera, 2002). Según Jessor (1993), la adolescencia es un período donde se busca la propia identidad y la independencia de los padres (Havinghurst, 1984; Steinberg \& Silverberg, 1986) y, paralelamente, se busca la aprobación de los compañeros de la misma edad en diferentes círculos sociales (Steinberg, 2007). Durante la adolescencia es posible relacionarse con ambientes sociales de forma más independiente que en la nińez, lo que posibilita el aprendizaje de comportamientos adaptativos o desadaptativos.

La educación formal impartida en las escuelas es de gran importancia en la adolescencia, debido a que permanecer dentro de una institución educativa durante esta etapa influye en el proceso de desarrollo y en el futuro (Tedesco \& López, 2004). También la escuela puede influir en la construcción de ciudadanos y en la adaptación a cambios tecnológicos y sociales; además, provee de mayores oportunidades personales de desarrollo económico y mejores condiciones de salud. En resumen, la educación brinda algunas herramientas que 
permiten satisfacción de necesidades básicas para el desarrollo integral del adolescente (Arguedas \& Jiménez, 2009).

La teoría de los sistemas del desarrollo postula que la interacción entre los aspectos biológicos, psicológicos y sociales del ser humano afecta el curso del desarrollo de modos diferentes (Cicchetti \& Rogosch, 1996). Estos aspectos pueden producir modos variados de respuesta que culminan en patrones adaptativos o desadaptativos de comportamiento (Cicchetti \& Dawson, 2002). Relacionado con lo anterior, subyacen dos principios derivados de la teoría general de los sistemas (Bertalanffy, 1962) que son básicos para la teoría de los sistemas del desarrollo: la equifinalidad y la multifinalidad (Cicchetti \& Rogosch, 1996). La equifinalidad implica que en cualquier sistema abierto una diversidad de trayectorias puede provocar el mismo resultado y la multifinalidad significa que cada componente puede funcionar diferencialmente dependiendo de la organización particular del sistema.

Masten y Coatsworth (1995), en el mismo marco conceptual, señalan que los individuos tienen propiedades de autoorganización y autorregulación que tienen la tarea de mantener su funcionamiento mientras se adaptan a los contextos donde se encuentran interactuando. La autorregulación es un proceso básico en la teoría del desarrollo que convierte a los individuos en agentes importantes de su propia evolución. La capacidad para controlar las emociones constituye un proceso importante para la adaptación humana; la regulación emocional abarca el iniciar una nueva respuesta o el alterar las respuestas emocionales a través de la acción de procesos regulatorios (Ochsner \& Gross, 2005). Investigaciones de tipo longitudinal realizadas desde la niñez a la adolescencia indican que el control emocional y conductual contribuye en la competencia académica y la salud psicológica (Sameroff \& Rosenblum, 2006). De esta manera, los procesos de autorregulación posibilitan la autorregulación del aprendizaje, el cual implica una capacidad superior de adaptación a las tareas, permite un aprendizaje autónomo y permanente, posibilitando que los estudiantes aumenten su rendimiento y éxito académico, utilizando diferentes 
estrategias, controlando y regulando aspectos de su cognición, motivación y conducta, mediando características contextuales y personales (Núñez, Solano, González-Pienda \& Rosário, 2006).

La teoría de los sistemas del desarrollo establece que existe continuidad en el desarrollo humano de la infancia hasta la edad adulta, pero la historia del desarrollo es modulada por las relaciones contextuales presentes y la diversidad de resultados posibles (Masten, 2006).

Existe una serie de situaciones que afectan el rendimiento académico de los jóvenes y ponen en riesgo su permanencia dentro de la institución educativa. Algunos autores plantean que las variables relacionadas al rendimiento académico pueden ser internas o externas, entendiéndose las internas como las características personales del estudiante y las externas como factores del contexto (Velázquez \& Rodríguez, 2006), de tal manera que el rendimiento académico constituye un fenómeno complejo en el cual convergen una gran cantidad de elementos que deben ser abordados. Entre las variables internas podemos destacar la resiliencia, la autorregulación y las metas educativas; mientras que como factores externos se encuentran las características negativas del vecindario donde habita el estudiante y los amigos de riesgo con los que se relaciona.

La resiliencia se define como la capacidad para presentar respuestas adaptativas ante condiciones adversas (Gaxiola \& Frías, 2007). La resiliencia es un constructo inferido que requiere el cumplimiento de tres características esenciales, la primera de ellas consiste en determinar la existencia de una amenaza al desarrollo; la segunda consiste en que la persona adquiera, a partir de sus interacciones psicológicas, la disposición para la resiliencia, esto es, la tendencia a la superación y posterior adaptabilidad ante las adversidades; y, por último, es necesario demostrar la competencia en una tarea del desarrollo de acuerdo con la edad y con la cultura (Gaxiola, Frías, Hurtado, Salcido \& Figueroa, 2011), por ejemplo un resultado académico.

La diversidad de resultados posibles en el desarrollo permite que algunos estudiantes superen los riesgos que se encuentran en algunas escuelas, evidenciando con ello el fenómeno de la resiliencia. Se entiende 
por factores de riesgo a aquellos atributos o variables que incrementan la probabilidad de que algunas personas con similares características desarrollen una problemática (Lucio, Rapp-Paglicci \& Rowe, 2011). Mientras, los factores protectores son aquellas condiciones o entornos capaces de favorecer el desarrollo de individuos o grupos, que pueden reducir los efectos de circunstancias desfavorables (Munist et al., 1998).

A pesar de que la resiliencia se manifiesta en individuos, esta constituye un proceso dinámico e interactivo entre las personas y los contextos, evidenciada en adaptaciones a condiciones adversas (Luthar, Cicchetti \& Becker, 2000). En América Latina, algunos autores han mencionado la resiliencia asociada a los logros educativos en estudiantes adolescentes (Villalta, 2010; Vinaccia, Quiceno \& San Pedro, 2007).

La autorregulación es otra variable individual asociada al rendimiento académico y se define como la capacidad de regular las propias emociones, la atención y el comportamiento (Block \& Block, 1980). Cuando las personas enfrentan demandas contextuales y personales necesitan controlar su atención, su conducta y sus estados afectivos (Zimmerman, 2000). El desarrollo de la capacidad autorregulatoria representa uno de los logros más importantes durante los primeros años de vida, dado que se relaciona con la competencia social, conductual y académica (Gilmore \& Cuskelly, 2005).

La autorregulación está asociada con el rendimiento académico (Valle et al., 2009), habiéndose reportado dicha relación en estudios longitudinales realizados por cuatro ańos con estudiantes adolescentes entre los 11 y 15 ańos de edad (Kim, Brody \& McBride, 2003). También se ha reportado una asociación entre la autorregulación y la resiliencia, la cual está mediada por la emoción positiva (Tugade \& Fredrickson, 2004).

Las metas, una variable individual de corte motivacional, constituyen representaciones cognoscitivas de los estados deseados (Austin \& Vancouver, 1996) que permiten la regulación, orientación, justificación de conductas y explican la toma de decisiones (Sanz de Acedo, Ugarte \& Lumbreras, 2003). Las investigaciones seńalan que las metas educativas se relacionan directamente con el rendimiento académico 
(Cunningham, Corprew \& Becker, 2009; Sánchez de Gallardo \& Pirela de Faría, 2006).

A nivel contextual, se ha encontrado que los vecindarios violentos influyen en los aspectos educativos. Por ejemplo, los vecindarios desorganizados con índices altos de pobreza, crimen y presencia de pandillas impactan de manera negativa a los estudiantes y a su rendimiento académico (Brunner \& Elacqua, 2004). El vecindario es una unidad de análisis importante porque puede afectar las condiciones sociales y psicológicas de las personas que habitan dentro de sus límites. La definición de vecindario incluye tanto los aspectos geográficos como los aspectos sociales de una comunidad particular (Garbarino, 1985), e implica tanto los apoyos sociales informales como las redes sociales (Garbarino, Kostelny \& Barry, 2002); por lo tanto, algunas de las amistades de los adolescentes son iniciadas y mantenidas en los vecindarios donde habitan.

Las amistades sirven al ser humano de múltiples maneras, entre ellas, la validación de los intereses, las esperanzas y los miedos, así como la provisión de apoyo y seguridad emocional (Rubin, 2004). Se ha reportado que las amistades positivas en la adolescencia se asocian con el envolvimiento y un rendimiento académico superior, mientras que las amistades negativas se relacionan con los problemas de conducta en la adolescencia (Burk \& Laursen, 2005) y con baja motivación académica (Nelson \& DeBacker, 2008).

A partir de la revisión anterior, el objetivo de la presente investigación fue probar dos modelos estructurales con variables contextuales e individuales del grupo resiliente y del grupo no resiliente, $y$ sus interrelaciones con el rendimiento escolar, partiendo de la hipótesis que establece que el grupo no resiliente presentará efectos significativos de las variables contextuales en el rendimiento académico, mientras que el grupo resiliente recibirá sus efectos significativos de las variables individuales en el rendimiento académico, moduladas por las disposiciones a la resiliencia y por las características autorregulatorias. 


\section{Método}

\section{Participantes}

La muestra fue seleccionada del listado oficial de alumnos de la institución, con las calificaciones respectivas en el semestre inmediato anterior. Del listado de alumnos, se eligieron las 20 calificaciones más altas y las 20 calificaciones más bajas de los semestres primero, tercero y quinto, de donde finalmente resultaron los 60 alumnos con las calificaciones más altas, así como los 60 alumnos con las calificaciones más bajas. En caso de que el alumno seleccionado estuviera dado de baja, sobre todo en el grupo de puntajes con menores calificaciones, se procedía a elegir al alumno siguiente de la lista. Al momento del estudio los alumnos se encontraban en los semestres segundo, cuarto y sexto respectivamente. Para conservar la confidencialidad del nombre de los alumnos, se les asignó un número de folio una vez que fueron llamados a la entrevista.

Los participantes fueron 120 estudiantes $(55.8 \%$ hombres y $44.2 \%$ mujeres), con un promedio 16.6 de ańos $(D E=1.81)$. El $33.3 \%$ de la muestra ( 40 alumnos) estaban inscritos en el segundo semestre, el $33.3 \%$ de la muestra cursaban el cuarto semestre ( 40 alumnos) y el $33.3 \%$ restante ( 40 alumnos) pertenecían al sexto semestre.

La mayoría de participantes $(75 \%)$ indicó que no trabajaba y se dedicaba exclusivamente a sus estudios. Por otra parte, un $68 \%$ de los alumnos reportaron que solamente en ocasiones les alcanza el ingreso familiar para cubrir sus necesidades básicas, lo que puede indicar que la mayoría de alumnos de la muestra tiene problemas económicos en la familia, que pueden asociarse negativamente con su rendimiento académico (Hernández, Márquez \& Palomar, 2006). El dato anterior contrasta con el $36.7 \%$ de alumnos que reportó que siempre les alcanza el ingreso familiar.

Los participantes conformaron dos grupos: un grupo de resiliencia formado por los 60 alumnos con las calificaciones más altas de la escuela, y un grupo de no resiliencia formado por los 60 alumnos con 
las calificaciones más bajas de la escuela. Lo anterior fue justificado a partir de la teoría que indica que la resiliencia implica una medida de competencia y de adaptabilidad ante los riesgos (Gaxiola et al., 2011). De cada uno de los semestres se seleccionaron 20 alumnos para el grupo de resiliencia (las 20 calificaciones más altas) y 20 alumnos para el grupo de no resiliencia (las 20 calificaciones más bajas), obteniendo en total 60 alumnos para el grupo de resiliencia y 60 alumnos para el grupo de no resiliencia.

\section{Instrumentos}

Inventario de datos sociodemográficos: se construyó este inventario con varias escalas que contenía primeramente los datos demográficos de los estudiantes como edad, sexo, si trabaja, grado educativo del padre y la madre, y el ingreso familiar alcanza para cubrir las necesidades básicas. Se incluyeron también escalas que midieron las metas educativas, la resiliencia, la autorregulación, las características del vecindario y las características de riesgo de los amigos.

Escala sobre Metas para Adolescentes (Sanz de Acedo, Ugarte \& Lumbreras, 2003): mide siete tipos de metas, pero se seleccionaron únicamente los reactivos relacionados con aspectos educativos para los fines del presente estudio. La consistencia interna de la escala obtuvo un valor de .92 y el factor metas educativas resultó con un valor de alfa de Cronbach de .84. La escala utilizada fue tipo Likert de 5 puntos, donde el participante respondió el grado de importancia que tiene para él cada afirmación de los reactivos, desde nada importante hasta muy importante.

Inventario de Resiliencia IRES (Gaxiola et al., 2011): está conformado por 20 ítems que evalúan siete dimensiones: actitud positiva, sentido del humor, perseverancia, religiosidad, autoeficacia, optimismo y orientación a la meta. Este inventario cuenta con cinco opciones de respuesta, donde 1 = nada y 5 = siempre. El inventario reporta un alfa de Cronbach de .93 en la escala total y valores de alfa que van de .65 a .95 para cada uno de sus factores. 
Behavior Rating Inventory of Executive Functions (BRIEF) (Gioia, Isquith, Retzlaff \& Espy, 2002): se utilizó la versión breve en español que contiene 30 preguntas que miden los problemas de regulación general ante diversas situaciones cotidianas, con siete opciones de respuesta que van desde $0=$ nunca hasta 6 = casi siempre. Se han reportado valores alfa de Cronbach de .90 para dicho instrumento (Gaxiola \& Frías, 2010).

Con el fin de evaluar el ambiente negativo del vecindario se emplearon 9 reactivos que han sido utilizados en otras investigaciones para registrar la percepción de los estudiantes acerca de este contexto (Frías, Fraijo \& Cuamba, 2008). La escala es de tipo Likert, donde $1=$ Nada y $5=$ Demasiado. La confiabilidad reportada por sus autores varía entre .78 y .80 .

Las características de riesgo de los amigos se evaluó a través de una escala elaborada especialmente para el estudio, donde se señala la frecuencia en que los amigos presentan comportamientos tales como consumir drogas, faltar a clases, participar en peleas o riñas, entre otras conductas disruptivas. La escala está conformada por 8 reactivos con 5 opciones de respuesta que van de Siempre a Nunca. La escala de amigos de riesgo obtuvo un valor alfa de .84 en el estudio piloto.

El rendimiento académico se midió con el promedio oficial individual de las calificaciones escolares en el semestre inmediato anterior, obtenido a través del listado proporcionado por la escuela.

\section{Procedimiento}

Se solicitaron los permisos respectivos para la investigación al director de la escuela. Previo consentimiento informado, se aplicó una batería de pruebas a una muestra de 120 alumnos de la preparatoria de Hermosillo, Sonora, con los más bajos puntajes de suficiencia académica, según la prueba ENLACE (SEP, 2010b). Los datos obtenidos fueron recogidos en el programa estadístico SPSS 17. Se realizaron los cálculos de las estadísticas descriptivas de las variables demográficas y posteriormente se sumaron los ítems de las escalas y resultaron índices. Se obtuvieron las alfas de todas las escalas y se analizaron 
las correlaciones entre los índices teóricos. Posteriormente los datos fueron analizados utilizando ecuaciones estructurales (Bentler, 2006), donde se probaron dos modelos estructurales, uno para el grupo de resiliencia y otro para el grupo de no resiliencia.

Para determinar la pertinencia del modelo propuesto se utilizaron indicadores de Bondad de Ajuste. La Bondad de Ajuste es un indicador de la pertinencia de una teoría de relaciones entre variables, dada por la correspondencia entre el modelo que la representa y los datos utilizados para probar esa teoría (Corral, Frías \& González, 2001). Para medir la Bondad de Ajuste del modelo existen dos tipos de indicadores: el indicador estadístico Chi-Cuadrado $\left(X^{2}\right)$ que determina la diferencia entre el modelo teórico propuesto y un modelo saturado formado por las relaciones entre todas las variables. Si el modelo teórico es pertinente, este no es diferente del saturado, por lo que el estadístico Chi-Cuadrado tendrá un valor alto y no significativo $(p>.05)$. Otros estadísticos que se usaron son los indicadores prácticos que consisten en una serie de estadísticos derivados del Chi-Cuadrado, que controlan el efecto del número de sujetos sobre la significatividad de la comparación. Se espera que los indicadores prácticos presenten valores cercanos a 1.0 para considerar al modelo con buena Bondad de Ajuste. De acuerdo con Bentler (2006), son ejemplos de dichos indicadores el Índice Bentler-Bonett de Ajuste Normado (IBAN) y el Índice BentlerBonett de Ajuste No Normado (IBANN).

\section{Resultados}

La Tabla 1 resume los datos sobre la escolaridad de los padres y madres de la muestra. Los alumnos encuestados reportaron que la mayoría de las madres (45.4\%) y de los padres (37.4\%) tuvieron la educación secundaria concluida, lo cual implica que sus progenitores, en promedio, tenían menor educación que ellos al momento de levantar los datos. Se ha reportado que a mayor escolaridad de los padres mayor rendimiento de los alumnos (Porcel, Dapozo \& López, 2010). 


\section{Tabla 1}

Escolaridad de los padres de la muestra

\begin{tabular}{lrrrr}
\hline & $\begin{array}{c}\text { Nivel educativo } \\
\text { mamá }\end{array}$ & \multicolumn{2}{c}{$\begin{array}{c}\text { Nivel educativo } \\
\text { papá }\end{array}$} \\
\hline Ninguna & $f$ & \multicolumn{1}{c}{$\%$} & \multicolumn{1}{c}{$f$} & $\%$ \\
Primaria & 3 & 2.5 & 4 & 3.4 \\
Secundaria & 21 & 17.6 & 25 & 21.7 \\
Preparatoria & 28 & 45.4 & 43 & 37.4 \\
Universidad & 13 & 10.9 & 15 & 13.0 \\
\hline
\end{tabular}

En la Tabla 2 se muestran los valores alfa de las escalas utilizadas en la evaluación y se muestra que todas las alfas son aceptables, dado que fueron superiores a 0.60 (Nieva \& Sorra, 2003).

\section{Tabla 2}

Valores Alfa de Cronbach de las escalas del estudio

\begin{tabular}{ll}
\hline Escalas & $\alpha$ \\
\hline Amigos de riesgo & .79 \\
Características negativas del vecindario & .82 \\
Metas educativas & .81 \\
Resiliencia & .89 \\
Autorregulación & .93 \\
\hline
\end{tabular}

La Tabla 3 muestra las correlaciones de Pearson del grupo de no resiliencia, sobresaliendo las relaciones significativas y positivas entre los amigos de riesgo y el vecindario negativo $(r=.33, p=.05)$, mientras que los amigos de riesgo y la autorregulación resultaron con una correlación negativa $(r=-.46, p=.01)$, así como los amigos de riesgo y las metas educativas $(r=-.30, p=.05)$.P por otra parte, las metas educativas se correlacionaron negativamente con las características negativas 
de los vecindarios $(r=-.42, p=.01)$. Lo anterior muestra las tendencias del grupo de no resiliencia a ser afectado por las variables contextuales. En este grupo el rendimiento académico no se correlacionó de manera significativa con ninguna variable.

\section{Tabla 3}

Correlaciones de Pearson del grupo de no resiliencia

\begin{tabular}{lcccccc}
\hline & $\begin{array}{c}\text { Pro- } \\
\text { medio }\end{array}$ & $\begin{array}{c}\text { Amigos } \\
\text { de riesgo }\end{array}$ & $\begin{array}{c}\text { Vecindario } \\
\text { negativo }\end{array}$ & $\begin{array}{c}\text { Metas } \\
\text { educativas }\end{array}$ & $\begin{array}{c}\text { Resi- } \\
\text { liencia }\end{array}$ & $\begin{array}{c}\text { Autoregu- } \\
\text { lación }\end{array}$ \\
\hline Amigos de riesgo & -- & 1 & & & & \\
Vecindario & -- & $.33^{* *}$ & 1 & & & \\
$\begin{array}{l}\text { negativo } \\
\text { Metas educativas }\end{array}$ & -- & $-.30^{*}$ & $-.42^{* *}$ & 1 & & \\
Resiliencia & -- & -- & -- & $.59^{* *}$ & 1 & \\
Autorregulación & -- & $-.46^{* *}$ & $-.32^{*}$ & $.36^{* *}$ & $.32^{* *}$ & 1 \\
\hline
\end{tabular}

Nota. ${ }^{*} p \leq .05$ (bilateral) ${ }^{* *} p \leq .01$ (bilateral).

Las correlaciones de Pearson del grupo de resiliencia en la Tabla 4 mostraron un patrón diferente, dado que no se encontró asociación significativa entre los amigos de riesgo y otras variables contextuales como las características negativas del vecindario. El grupo de resiliencia obtuvo correlaciones significativas entre las metas educativas y la resiliencia $(r=.44, p=.01)$, y la autorregulación con la resiliencia $(r=.48$, $p=.01)$. Contrario al grupo de no resiliencia, la variable metas educativas correlacionó significativa y positivamente con el rendimiento académico $(r=.33, p=.01)$, así como la variable resiliencia $(r=.33$, $p=.05)$. El patrón anterior de correlaciones mostró una tendencia del grupo de resiliencia a ser afectado positivamente por las variables individuales. 


\section{Tabla 4}

Correlaciones de Pearson del grupo de resiliencia

\begin{tabular}{lcccccc}
\hline & $\begin{array}{c}\text { Pro- } \\
\text { medio }\end{array}$ & $\begin{array}{c}\text { Amigos } \\
\text { de riesgo }\end{array}$ & $\begin{array}{c}\text { Vecindario } \\
\text { negativo }\end{array}$ & $\begin{array}{c}\text { Metas } \\
\text { educativas }\end{array}$ & $\begin{array}{c}\text { Resi- } \\
\text { liencia }\end{array}$ & $\begin{array}{c}\text { Autorre- } \\
\text { gulación }\end{array}$ \\
\hline Amigos de riesgo & -- & 1 & & & & \\
Vecindario & -- & -- & 1 & & & \\
negativo & & -- & -- & 1 & 1 & \\
Metas educativas & $.33^{* *}$ & - & -- & $.44^{* *}$ & 1 & 1 \\
Resiliencia & $.26^{*}$ & -- & $-.26^{*}$ & -- & $.48^{* *}$ & 1 \\
Autorregulación & -- & -- & &
\end{tabular}

Nota: ${ }^{*} p \leq .05$ (bilateral); ${ }^{* *} p \leq .01$ (bilateral).

Las correlaciones encontradas tanto en el grupo de resiliencia como en el grupo de no resiliencia establecieron el sustento empírico para probar la trayectoria de dichas relaciones en modelos de ecuaciones estructurales (Bentler, 2006).

El modelo estructural del grupo de no resiliencia, presentado en la Figura 1, muestra las trayectorias del rendimiento académico con sus interrelaciones entre las variables contextuales e individuales. Las trayectorias indican que el vecindario de riesgo predijo positiva y significativamente a los amigos de riesgo (coeficiente estructural $=.33$ ). Los amigos de riesgo, a su vez, predijeron negativamente a la autorregulación (coeficiente estructural $=-.40$ ). La autorregulación predijo positivamente a la resiliencia (coeficiente estructural = -.32) y la resiliencia predijo también positivamente a las metas educativas (coeficiente estructural $=-.55)$. Mientras tanto las metas fueron afectadas negativamente por el vecindario de riesgo (coeficiente estructural $=-.38$ ). No se encontraron relaciones directas significativas entre el vecindario de riesgo y la autorregulación, ni tampoco entre la autorregulación y las metas educativas. Como era de esperarse, a partir de los resultados de las correlaciones, no existe relación significativa entre las metas educativas del grupo de no resiliencia y el rendimiento académico. 
Los indicadores de bondad de ajuste del modelo de no resiliencia (BBNFI, BBNNFI y $C F I$ ) fueron cercanos a 1.0 , mientras que el Chi-Cuadrado del modelo fue alto y no significativo $\left(X^{2}=80.1\right.$, $p=.46$ ), con un Residuo de Cuadrados Mínimos (RMSEA) de .00. Esto demuestra que el modelo teórico se ajusta a los datos, y que el modelo sintético no es diferente significativamente del modelo saturado el cual está formado por las relaciones entre todas las variables (Bentler, 2006; Corral, Frías \& González, 2001). El modelo del grupo de no resiliencia no explicó nada del rendimiento académico, aunque en las metas educativas resultó una $R^{2}=.53$.

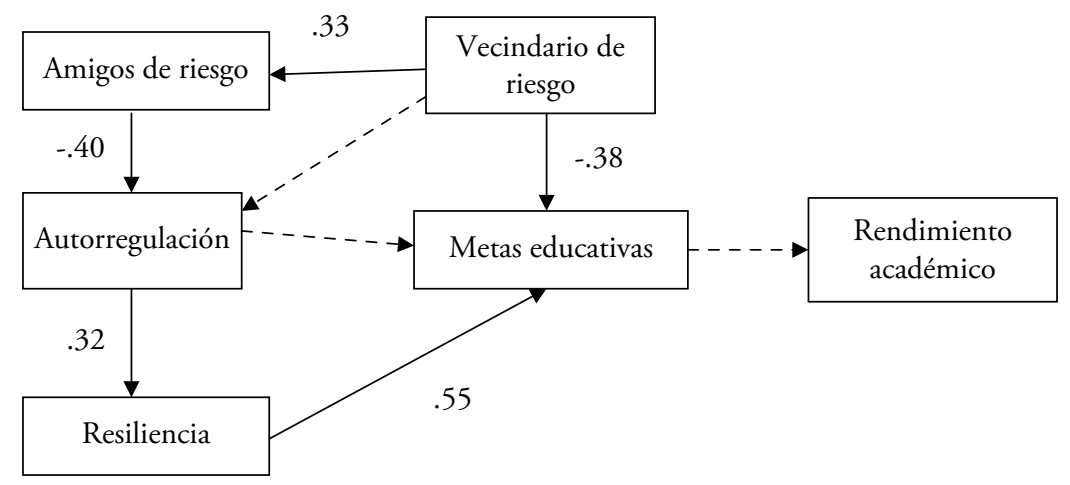

$$
X^{2}=80.1,15 \mathrm{gl}, p=.462 \mathrm{BBNFI}=.91 \mathrm{BBNNFI}=1.0 \mathrm{CFI}=1.0 \mathrm{RMSEA}=.00
$$

Figura 1. Modelo de trayectorias del grupo de no resiliencia.

La Figura 2 muestra las trayectorias del grupo de resiliencia, donde la autorregulación predijo positiva y significativamente la resiliencia (coeficiente estructural $=.47$ ), la resiliencia predijo positivamente las metas educativas (coeficiente estructural $=-.43$ ), y las metas educativas también predijeron positivamente al rendimiento académico (coeficiente estructural $=-.27)$. Al probarse los efectos del vecindario en los amigos de riesgo, y de esta última variable en la autorregulación, la resiliencia y las metas educativas, no se encontraron efectos significativos. 
Los indicadores de bondad de ajuste del modelo de resiliencia (BBNFI, BBNNFI y CFI) fueron cercanos a 1.0, mientras que el indicador Chi-Cuadrado del modelo fue alto y no significativo $\left(X^{2}=47.2\right.$, $p=.41$ ), con un Residuo de Cuadrados Mínimos (RMSEA) de .02. Esto significa que el modelo teórico se ajusta a los datos, y el modelo sintético no es diferente significativamente del modelo saturado, formado por las relaciones entre todas las variables (Bentler, 2006; Corral, Frías \& González, 2001). El rendimiento académico de este grupo resultó con una varianza explicada del $13 \%\left(R^{2}=.13\right)$.

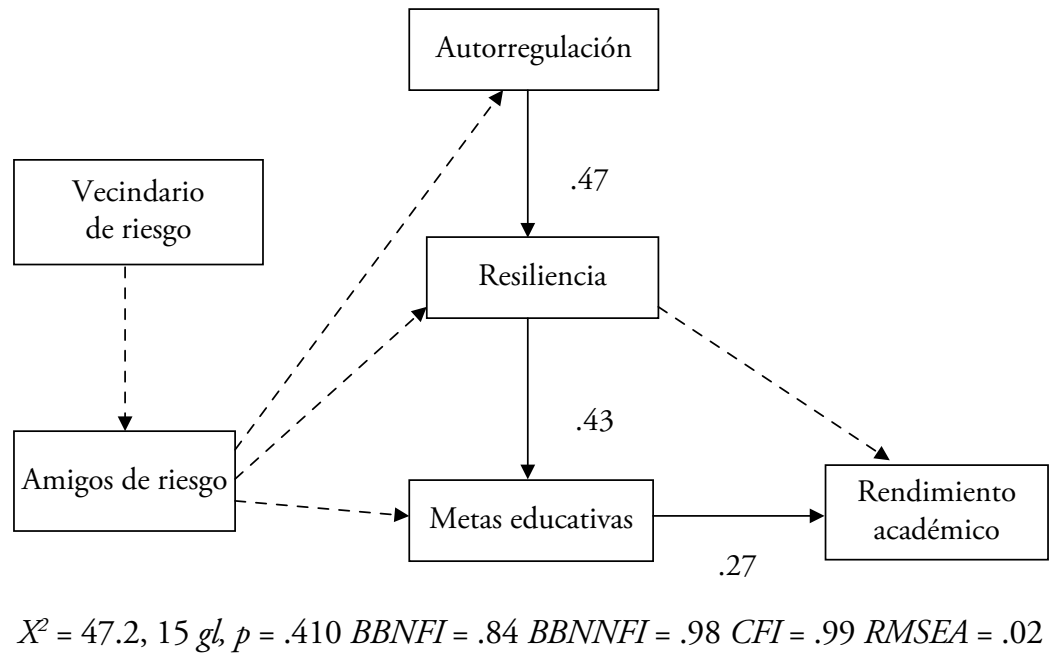

Figura 2. Modelo de trayectorias del grupo de resiliencia.

\section{Discusión}

La varianza explicada en las metas educativas del modelo de no resiliencia (Figura 1) indican que, aunque este grupo reportó metas educativas, no se relacionaron significativamente con su rendimiento académico, en este caso porque estaba representado por sus bajas calificaciones. Lo anterior puede ser porque en el modelo existe una relación 
significativa más relevante, constituida por el efecto negativo del vecindario de riesgo sobre las metas educativas. A partir de lo anterior, el modelo de no resiliencia no explicó nada del rendimiento académico, sin embargo, lo importante del presente modelo radica en la evidencia que presentó con respecto a los efectos de las variables contextuales del vecindario de riesgo y los amigos de riesgo, en los procesos de autorregulación de los alumnos con bajas calificaciones, que pueden en un momento dado ser moduladas por la disposición individual a la resiliencia.

En el modelo estructural del grupo de resiliencia (Figura 2) sobresale que no se encontraron influencias significativas del vecindario de riesgo, ni tampoco de los amigos de riesgo, en las variables de autorregulación, resiliencia o en las metas educativas. En este grupo, contrario al grupo de no resiliencia, la variable autorregulación mediada por la resiliencia predijo a las metas educativas, las cuales predijeron positivamente al rendimiento académico. Por tal razón, el modelo de resiliencia presentó una varianza explicada del rendimiento académico del 13\% que, aunque es baja, al menos explicó una proporción del rendimiento académico de esta parte de la muestra.

Al comparar los dos modelos resultantes, los alumnos pertenecientes al grupo de no resiliencia (aquellos con las más bajas calificaciones) se afectaron más por la influencia de la variables contextuales, mientras que los pertenecientes al grupo de resiliencia (los alumnos con las más altas calificaciones) fueron mas influidos por las variables individuales probadas. Es decir, el grupo de resiliencia no fue predicho de manera significativa por las variables contextuales negativas que influyeron en la trayectoria del grupo de no resiliencia. En el caso del grupo de resiliencia, los efectos de las variables contextuales negativas fueron amortiguados por las características individuales de autorregulación y resiliencia, las cuales predijeron a las metas educativas que finalmente afectaron al rendimiento académico. Con base en lo anterior, se sustenta el principio que indica que la resiliencia es un fenómeno basado en las diferencias individuales (Rutter, 2007), pues en algunos se establece la disposición individual para la resiliencia y se amortiguan los efectos de los riesgos, modulados por los procesos de autorregulación. De esta 
manera, los resultados de la resiliencia como proceso psicológico posibilitan la adaptabilidad del ser humano frente a los retos que presentan los entornos o circunstancias adversas, donde la autorregulación tiene un papel central. La resiliencia constituye, entonces, la inferencia tanto del proceso psicológico como del resultado adaptativo.

Los hallazgos comprueban la hipótesis de la presente investigación y fortalecen el principio de multifinalidad de la teoría de los sistemas del desarrollo utilizado en la investigación (Cicchetti \& Rogosch, 1996) y el papel de la autorregulación en el desarrollo psicológico (Masten \& Coatsworth, 1995), pero se requieren estudios futuros con otras poblaciones y en otros contextos que brinden mayor sustento al hallazgo anterior. Sobre todo, se proponen investigaciones de corte longitudinal que disminuyan los sesgos posibles como el de la presente investigación, donde se utiliza el autoreporte como medio para la obtención de datos (Aguilar, Valencia \& Romero, 2004).

La influencia de las condiciones físicas de inseguridad del vecindario en el grupo de no resiliencia puede ser explicada debido a que algunos adolescentes, conforme llegan a los estudios de preparatoria, son más susceptibles de ser impactados por dichas condiciones contextuales (Woolley et al., 2008). Lo anterior puede estar basado en la importancia que adquieren los contextos fuera del hogar conforme se llega a la adolescencia (Steinberg, 2007). En los vecindarios donde existe violencia e infraestructura física inadecuada, es más probable que los adolescentes se relacionen con amistades que los alejen de sus actividades académicas. Hickey (2003) señala que el aprendizaje implica la participación del contexto donde el individuo se relaciona, sobre todo, porque el conocimiento adquirido es valorado y tiene alguna utilidad en dicho escenario. La relación entre las características del vecindario y el rendimiento académico ha sido encontrada en estudiantes de una secundaria en riesgo, ubicada en un contexto similar al del presente estudio (Gaxiola \& González, en prensa). La diferencia con el grupo de resiliencia puede ser el mecanismo de autorregulación que opera en estos alumnos, sin la interferencia de las variables contextuales. 
La autorregulación, tanto en el modelo de resiliencia como en el modelo de no resiliencia, constituye un mecanismo regulador. Shapiro y Schuartz (2000) señalan que la autorregulación es un componente central de la resiliencia y la competencia en todas las áreas del desarrollo humano. La autorregulación posibilita a los jóvenes que se relacionen menos con amistades negativas o se involucren menos en conducta antisocial (Bandura, Barbaranelli, Caprara \& Pastorelli, 1996). Debido a lo anterior, se ha encontrado que los procesos de autorregulación de la conducta se han relacionado con el rendimiento académico en otras investigaciones (Kim, Brody \& McBride, 2003; Valle et al., 2009). En el presente estudio, a pesar de la actuación del proceso de autorregulación en los adolescentes del grupo de no resiliencia, este no fue lo suficientemente significativo como para contrarrestar el efecto de las variables contextuales relacionadas con el habitar en vecindarios negativos, o estar bajo la influencia de los amigos de riesgo.

La relación entre la autorregulación y la resiliencia ha sido reportada en otras investigaciones realizadas con estudiantes de secundaria en riesgo de la región (Gaxiola \& González, en prensa), lo cual implica que este mecanismo representa un proceso regulador importante en los estudiantes de edad adolescente. En la medida que los procesos autorregulatorios predicen las disposiciones individuales a la resiliencia, se aportan datos sobre la posible base psicobiológica de algunos mecanismos que participan en el fenómeno de la resiliencia (Rutter, 2007).

La resiliencia medida como disposiciones conductuales positivas (actitud positiva, sentido del humor, perseverancia, religiosidad, autoeficacia, optimismo y orientación a la meta) se ha relacionado con las metas educativas y con el rendimiento académico de estudiantes de secundaria (Gaxiola \& González, en prensa). Los datos encontrados permiten comprobar que dicha relación también se presenta en los alumnos pertenecientes al grupo de resiliencia de la muestra. Lo anterior puede explicarse debido a que algunos indicadores utilizados para la resiliencia corresponden con la capacidad de planeación y enfrentamiento de los problemas de la vida, que en el caso de los adolescentes 
muy probablemente se asocian con sus metas educativas. Este hallazgo requiere de mayor investigación.

Las metas o expectativas educativas predijeron únicamente el rendimiento académico en el grupo de resiliencia, probablemente por la presencia de amigos de riesgo en el grupo de no resiliencia, lo cual ha sido reportado en otras investigaciones (Cunningham, Corprew $\&$ Becker, 2009). En la medida que los dos grupos estudiados presentaron los mismos riesgos contextuales, es posible que el grupo de no resiliencia sea entrenado en la elección de compañeros más acordes con las actividades académicas y se fortalezca, al mismo tiempo, su resiliencia y autorregulación. Realizando tales actividades es posible que sean menos afectados por el vecindario de riesgo donde habitan y por los amigos de riesgo con los que conviven diariamente. El apoyo social recibido de amistades positivas puede afectar el rendimiento académico de los estudiantes en riesgo (Burk \& Laursen, 2005).

La autorregulación constituye una habilidad importante en el desarrollo humano relacionada con el rendimiento académico, y es posible que se apliquen programas para su desarrollo en nińos y adolescentes basados en la posibilidad del autocontrol de las emociones (Macklem, 2011). Por otra parte, existen programas para la promoción de la resiliencia por medio del entrenamiento en habilidades de afrontamiento y establecimiento de metas en la vida, incremento de apoyos sociales, entre otras acciones (Henderson \& Milstein, 2003). La aplicación de programas sistemáticos de atención a las variables asociadas al logro escolar, en las escuelas de educación media superior, sobre todo las que presentan mayores riesgos, puede mejorar el rendimiento académico de sus alumnos una vez que se valoren sus efectos con estudios diagnósticos previos. 


\section{Referencias}

Aguilar, V. J., Valencia, C. A. \& Romero, S. P. (2004). Estilos parentales y desarrollo psicosocial en estudiantes de bachillerato. Revista Mexicana de Psicología, 21(2), 119-129.

Arguedas, N. I. \& Jiménez, S. F. (2009). Permanencia en la educación secundaria y su relación con el desarrollo positivo durante la adolescencia. REICE, Revista Electrónica Iberoamericana sobre la Calidad, Eficacia y Cambio en Educación, 7, 50-65.

Austin, J. T. \& Vancouver, J. B. (1996). Goal constructs in psychology: Structure, process and content. Psychological Bulletin, 120(3), 338-375.

Bandura, A., Barbaranelli, C., Caprara, G. V. \& Pastorelli, C. (1996). Mechanisms of moral disengagement in the exercise of moral agency. Journal of Personality and Social Psychology, 71, 364-374. Bentler, P. M. (2006). EQS 6 Structural Equations Program Manual. Encino, CA: Multivariate Software.

Bertalanffy, L. V. (1962). Modern theories of development: An introduction to theoretical biology. Nueva York: Harper \& Row. (Trabajo original publicado en 1933)

Block, J. H. \& Block, J. (1980). The role of ego-control and egoresiliency in the organization of behavior. En W. A. Collins (Ed.), The Minnesota Symposia on Child Psychology (Vol. 13, pp. 39-101). Hillsdale, NJ: Erlbaum.

Brunner, J. J. \& Elacqua, G. (2004). Factores que inciden en una educación efectiva. Evidencia internacional. Revista de la Educación, 139-140, 1-11.

Burk, W. J. \& Laursen, B. (2005). Adolescent perceptions of friendship and their association with individual adjustment. International Journal of Behavioral Development, 29, 156-164.

Cascón, I. (2000). Análisis de las calificaciones escolares como criterio de rendimiento académico. Recuperado de http:/www3.usal.es/ inico/investigacion/ 
Cicchetti, D. \& Dawson, G. (2002). Multiple levels of analysis. Development and Psychopathology, 14(3), 417-666.

Cicchetti, D. \& Rogosch, F. A. (1996). Equifinality and multifinality in developmental psychopathology. Development and Psychopathology, 8, 597-600.

Corral, V., Frías, M. \& González, D. (2001). Análisis cuantitativo de variables latentes. Hermosillo, México: UniSon.

Cunningham, M., Corprew, C. S. \& Becker, J. E. (2009). Associations of future expectations, negative friends, and academic achievement in high-achieving African American adolescents. Urban Education, 44(3), 280-296.

De Hoyos, R., Espino, J. M. \& García, V. (2010). Determinantes del logro escolar en México: primeros resultados utilizando la prueba ENLACE media superior. México D. F.: Secretaría de Educación Media Superior.

Frías, M., Fraijo, B. \& Cuamba, N. (2008). Problemas de conducta en niños víctimas de violencia familiar: reporte de profesores. Estudos de Psicología, 13(1), 3-11.

Frydenberg, E. (1997). Adolescent coping. Londres: Routhledge.

Garbarino, J. (1985). An ecological approach to child maltreatment. En L. E. Pelton (Ed.), The social context of child abuse and neglect (pp. 228-267). Nueva York: Human Sciences.

Garbarino, J., Kostelny, K. \& Barry, F. (2002). Neighborhood-based programs. En P. K. Trickett \& C. J. Schellenbach (Eds.), Violence against children in the family and the community (pp. 287-314). Washington DC: American Psychological Association.

Gaxiola, R. J. \& Frías, M. (2007). Los factores protectores y la adaptabilidad al abuso infantil: un estudio con madres mexicanas. Revista Mexicana de Psicología, 222-223.

Gaxiola, R. J. \& Frías, M. (2010). Child maltreatment, self-regulation and parenting. En M. Frías \& V. V. Corral (Eds.), Bio-psychosocial perspectives on interpersonal violence (pp. 101-121). Nueva York: Nova Science. 
Gaxiola, R. J., Frías, M., Hurtado A. M., Salcido, N. L. \& Figueroa, F. M. (2011). Validación del Inventario de Resiliencia (IRES) en una población del noroeste de México. Enseñanza e Investigación en Psicología, 16(1), 73-83.

Gaxiola, R. J. \& González, L. S. (en prensa). Predictores del rendimiento académico y resiliencia de adolescentes de nivel secundaria. En J. Palomar \& J. Gaxiola (Eds.), Estudios de resiliencia en América Latina. México D. F.: Manual Moderno.

Gilmore, L. A. \& Cuskelly, M. (2005) The measurement of self-regulation from ages 2 to 8 . En L. A. Gilmore \& M. Cuskelly (Eds.), Proceedings of the $40^{\text {th }}$ APS Annual Conference, Melbourne, Australia. Recuperado de http://eprints.qut.edu.au

Gioia, G., Isquith, P., Retzlaff, P. D. \& Espy, K. A. (2002). Confirmatory factor analysis of the Behavioral Rating Inventory of Executive Function [BRIEF] in a clinical sample. Child Neuropsychology, 8, 294-257.

Havinghurst, R. J. (1984). Developmental tasks and education. Nueva York: David McKay.

Henderson, N. \& Milstein, M. M. (2003). Resiliencia en la escuela. Barcelona: Paidós.

Hernández, J., Márquez, A. \& Palomar, J. (2006). Factores asociados con el desempeño académico en el EXANI-I Zona Metropolitana de la Ciudad de México 1996-2000. Revista Mexicana de Investigación Educativa, 11(29), 547-581.

Hickey, D. T. (2003). Engaged participation versus marginal nonparticipation: A stridently sociocultural approach to achievement motivation. The Elementary School Journal, 103(4), 401-429. Recuperadodehttp://www.enlace.sep.gob.mx/gr/docs/historico/ 26_Sonora_ENLACE2010.pdf

Jessor, R. (1993). Successful adolescent development among youth in high-risk settings. American Psychologist, 48(2), 117-126.

Jiménez, M. (2000). Competencia social: intervención preventiva en la escuela. Infancia y Sociedad, 24, 21-48. 
Kim, S., Brody, G. H. \& McBride M. V. (2003). Longitudinal links between contextual risks, parenting, and youth outcomes in rural African American families. Journal of Black Psychology, 29(4), 359-377.

Lucio, R., Rapp-Paglicci, L. \& Rowe, W. (2011). Developing an additive risk model for predicting academic index: School factors and academic achievement. Journal of Child Adolescence and Social Work, 28, 153-173.

Luthar, S. S., Cicchetti, D. \& Becker, B. (2000). The construct of resilience: A critical evaluation and guidelines for future work. Child Development, 71, 543-562.

Macklem, G. L. (2011). Importance of emotion regulation training for children and adolescents. En I. Nyklicek, A. Vingerhoets \& M. Zeelenberg (Eds.), Emotion regulation and well-being: A view from different angles (pp. 85-106). Nueva York: Springer.

Masten, A. S. (2006). Developmental psychopathology: Pathways to the future. International Journal of Behavioral Development, 30(1), 47-54.

Masten, A. S. \& Coatsworth, J. D. (1995). Competence, resilience, and psychopathology. En D. Cicchetti \& D. Cohen (Eds.), Developmental psychopathology: Vol. 2. Risk, disorder, and adaptation (pp. 715-752). Nueva York: Wiley.

Munist, M., Santos, H., Kotliarenco, M. A., Suárez, O. E., Infante, F. \& Grotberg, E. (1998). Manual de identificación y promoción de la resiliencia en niños y adolescentes. Washington, D. C.: Organización Panamericana de la Salud y Organización Mundial de la Salud.

Nelson, R. M. \& DeBacker, T. K. (2008). Achievement motivation in adolescents: The role of peer climate and best friends. Journal of Experimental Education, 76(2), 170-189.

Nieva, V. F. \& Sorra, J. (2003). Safety culture assessment: A tool for improving patient safety in healthcare organizations. Quality Safe Health Care, 12 (supl), ii17-ii23. 
Núñez, J. C., Solano, P, González-Pienda, J. A. \& Rosário, P. (2006). El aprendizaje autorregulado como medio y meta de la educación. Papeles del Psicólogo, 3(27), 139-146.

Ochsner, K. N. \& Gross, J. J. (2005). The cognitive control of emotion. Trends in Cognitive Sciences, 9(5), 242-249.

Pizarro, R. \& Clarck, S. (1998). Currículo del hogar y aprendizajes educativos. Interacción versus estatus. Revista de Psicología de la Universidad de Chile, 7, 25-33.

Porcel, E., Dapozo, G. \& López, M. (2010). Predicción del rendimiento académico de alumnos de primer año de la FACENA (UNNE) en función de su caracterización socioeducativa. Revista Electrónica de Investigación Educativa, 12(2), 1-21.

Rubin, K. H. (2004). Three things to know about friendship. Newsletter International Society for the Study of Behavioral Development, 46(2), 5-7.

Rutter, M. (2007). Resilience, competence, and coping. Child Abuse \& Neglect, 3, 205-209.

Sameroff, A. J. \& Rosenblum, K. L. (2006). Psychosocial constraints on the development of resilience. Annals of the New York Academy of Sciences, 1094, 116-124.

Sánchez de Gallardo, M. \& Pirela de Faría, L. (2006). Motivaciones sociales y rendimiento académico en estudiantes de educación. Revista de Ciencias Sociales, 12(1), 1-20.

Sanz de Acedo, L. M., Ugarte, M. D. \& Lumbreras, B. M. (2003). Desarrollo y validación de un cuestionario de metas académicas para adolescentes. Psicothema, 3(15), 493-499.

Secretaría de Educación Pública. (2010a). ENLACE Básica y Media Superior, Datos Nacionales, 2010. Recuperado de http://enlace. sep.gob.mx/ms/?p=estadisticas 2010

Secretaría deEducación Pública. (2010b). ENLACE Básicay Media Superior, Estado de Sonora, 2010. Recuperado de http://www.enlace. sep.gob.mx/gr/docs/historico/26_Sonora_ENLACE2010.pdf

Shapiro, S. \& Schuartz, G. (2000). The role of intention in self-regulation. Toward intentional systemic mindfulness. En M. Boekaerts, 
P. Pintrich \& M. Zeidner (Eds.), Handbook of self-regulation. San Diego, CA: Elsevier Academic Press.

Steinberg, L. (2007). Risk taking in adolescence: New perspectives from brain and behavioral science. Current Directions in Psychological Science, 16, 55-59.

Steinberg, L. \& Silverberg, S. B. (1986). The vicissitudes of autonomy in early adolescence. Child Development, 57, 841-851.

Tedesco, J. C. \& López, N. (2004). Algunos dilemas de la educación secundaria en América Latina. REICE, Revista Electrónica Iberoamericana sobre Calidad, Eficacia y Cambio en Educación, 2(1), 55-69.

Tugade, M. M. \& Fredrickson, B. L. (2004). Resilient individuals use positive emotions to bounce back from negative emotional experiences. Journal of Personality and Social Psychology, 86(2), 320-333.

Valle, A., Núñez, J. C., Cabanach, R. G., Rodríguez, S., GonzálezPienda, J. A. \& Rosário, P. (2009). Perfiles motivacionales en estudiantes de secundaria: análisis diferencial en estrategias cognitivas, estrategias de autorregulación y rendimiento académico. Revista Mexicana de Psicología, 26, 113-124.

Vargas, T. E. \& Barrera, F. (2002). Adolescencia, relaciones románticas y actividad sexual: una revisión. Revista Colombiana de Psicología, $11,115-134$.

Velázquez, T. L. \& Rodríguez, S. N. (2006). Rendimiento académico y contexto familiar en estudiantes universitarios. Enseñanza e Investigación en Psicología, 11(2), 255-270.

Villalta, P. M. (2010). Factores de resiliencia asociados al rendimiento académico en estudiantes de contextos de alta vulnerabilidad social. Revista Pedagógica, 31(88), 159-190.

Vinaccia, S., Quiceno, M. J. \& San Pedro, E. M. (2007). Resiliencia en adolescentes. Revista Colombiana de Psicología, 16, 139-146.

Woolley, M. E., Grogan-Kaylor, A., Gilster, M. E., Karb, R. A., Gant, L. M., Reischl, T. M. et al. (2008). Neighborhood social capital, poor physical conditions, and school achievement. Children and Schools, 30(3), 133-145. 
Zimmerman, B. J. (2000). Attaining self-regulation: A social cognitive perspective. En M. Boekaerts, P. R. Pintrich \& M. Zeidner (Eds.), Handbook of self-regulation (pp. 13-39). San Diego, CA: Elsevier Academic Press.

Recibido: 24 de junio, 2011 Aceptado: 25 de octubre, 2011 